\title{
AUMENTO DO RENDIMENTO DE TURBINA DE TOPO DE ALTO FORNO VIA APLICAÇÃO DE POLIMERO DISPERSANTE DE ALTO ESTRESSE *
}

\author{
Paulo Santiago ${ }^{1}$
}

Luis Paulo Zanin Armacollo²

Tarun Bera ${ }^{3}$

\section{Resumo}

Redução dos custos de operação e a produção de aço com menor impacto ambiental são diretrizes unânimes na siderurgia atual. A tecnologia das Turbinas de Recuperação de Topo (Top Recovery Turbines - TRT) é um aliado importante para seguir nessas direções. As TRTs produzem energia elétrica a partir do gás de alta pressão proveniente do topo dos Altos Fornos, gerando energia elétrica sem impacto em emissões. Durante a campanha de operação da turbina, a mesma pode perder eficiência operacional devido a, dentre outros, deposição sobre as palhetas. Com o intuito de melhorar o desempenho da turbina estabilizando sua geração durante toda sua campanha, foi desenvolvido um trabalho de dosagem de polímero dispersante na água de spray da turbina (para turbinas com tecnologia de água de spray na admissão do gás). A dosagem do polímero dispersante visa reduzir a deposição e incrustração nas palhetas, reduzindo assim a perda de performance durante a campanha causadas pelo incremento de massa nas palhetas. Os resultados alcançados em um teste de campo em uma siderúrgica no Brasil foram um incremento de $52 \%$ na geração durante a campanha onde o polímero dispersante foi aplicado. Com a aplicação do polímero, uma melhora significativa na confiabilidade e performance da turbina assim como a possibilidade da redução no consumo de água foram atingidos

Palavras-chave: Turbina de Topo; Alto Forno; Eficiência Energética; Redução TCO.

\section{DEPOSIT CONTROL WITH HIGH STRESS POLYMER PROGRAM IMPROVES ENERGY GENERATION IN BLAST FURNACE WET-TYPE TOP RECOVERY TURBINES}

\section{Abstract}

Operational cost reduction and to produce steel with less environmental impact are key targets for steel industry. Top Recovery Turbine (TRT) is one of the technologies going on this direction. TRT produces energy from blast furnace top high pressure gas, generating energy without emission impact. During the TRT campaign the operational efficiency can be reduced due to fouling and deposition over the blades. Looking to improve the turbine efficiency and its performance, stabilizing the power output during all the campaign, a chemical program dosing high stress polymer at the spray water was developed and tested (for wet-type turbines). The polymer application target was to reduce the fouling over the blades allowing the turbine to keep its best performance rates since the beginning of the campaign up to the end, reducing the lost in power outlet performance due to solids deposit. An increase of $52 \%$ on turbine generation was achieved on a field trial on a mill located in Brazil with this polymer application, with a significant improvement on reliability and turbine performance and the possibility to reduce water consumption by using reuse water for spray.

Keywords: Top Recovery Turbine, Blast Furnace, Energy Efficiency, TCO reduction.

1 Paulo Santiago, Engenheiro Químico, Gerente Senior de Marketing, Nalco Water, São Paulo Brasil

2 Luis Paulo Zanin Armacollo, Engenheiro Químico, Sr Marketing Associate, Nalco Water, Vitória Brasil

3 Tarun Bera, Ph.D. Química, Dr Staff Scientist, RD\&E, Nalco Water,Maharashtra - India 


\section{INTRODUÇÃO}

$\mathrm{Na}$ visão de melhorar os custos operacionais das indústrias siderúrgicas, um dos campos com potencial para tal é o da energia elétrica. Trabalhos com intuito de melhoria de eficiência energética são cada vez mais valorizados em todo os ramos, e dentro da siderurgia em especial. As usinas equipadas com altos fornos tem a possibilidade de recuperar o gás de topo dos altos fornos para produção direta de energia através das Turbinas de Topo. Estas turbinas têm por principio a geração direta de energia elétrica recuperando o gás gerado no alto forno, não necessitando de combustíveis para queima e evitando a geração de $\mathrm{CO} 2$ ou outros gases de efeito estufa, ou seja, uma geração limpa.

Existem dois tipos de turbina de topo: Wet Type e Dry Type. Este trabalho se aplica às turbinas de topo do tipo Wet Type. Este modelo de turbina usa água de spray que é adicionada na entrada da turbina junto com o gás de Alto Forno, antes do Stator Blade.

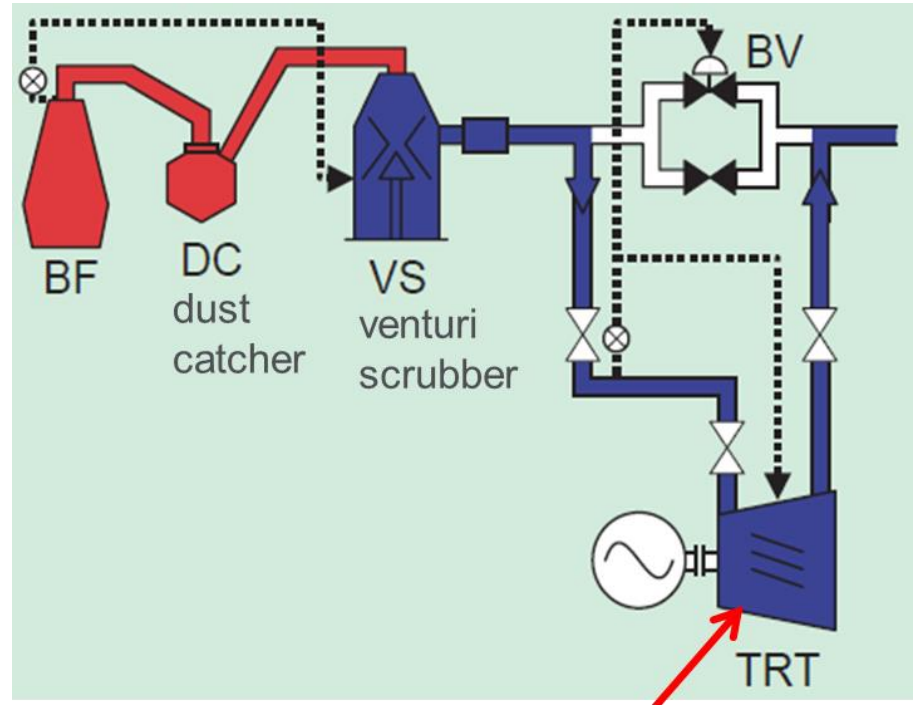

Figura 1. Esquema genérico de uma turbina de topo do tipo Wet-type

Um dos problemas encontrados na operação das Turbinas de Topo é a deposição nas palhetas do stator blade e da própria turbina, causados pela qualidade da água de spray e também pela qualidade do gás. Esta deposição de material causa distúrbios operacionais, de manutenção e redução da capacidade de geração da Turbina.

Com o intuito de minimizar os impactos da deposição e incrustração na turbina, este trabalho visa apresentar os resultados da aplicação de polímeros de alto estresse na água de spray destas turbinas de topo que reduzem a deposição de material nas palhetas, reduzindo assim o impacto na geração de energia e em sua manutenção.

\section{MATERIAIS E MÉTODOS}

Baseado em auditorias, pesquisas e enquetes realizadas com siderúrgicas ao redor do mundo, e na análise de água utilizada para o spray, a Nalco desenvolveu um programa químico com moléculas de resistência a altos padrões de estresse (temperatura, pressão, vazão, turbulência) com capacidade dispersante e antiincrustante. 
$\mathrm{Na}$ aplicação industrial aqui apresentada, duas qualidades de água distintas estavam sendo utilizadas como água de spray da turbina: Água Industrial e Água de Lavagem de gases do Alto Forno. A diferença de qualidade das fontes de água é demonstrada abaixo:
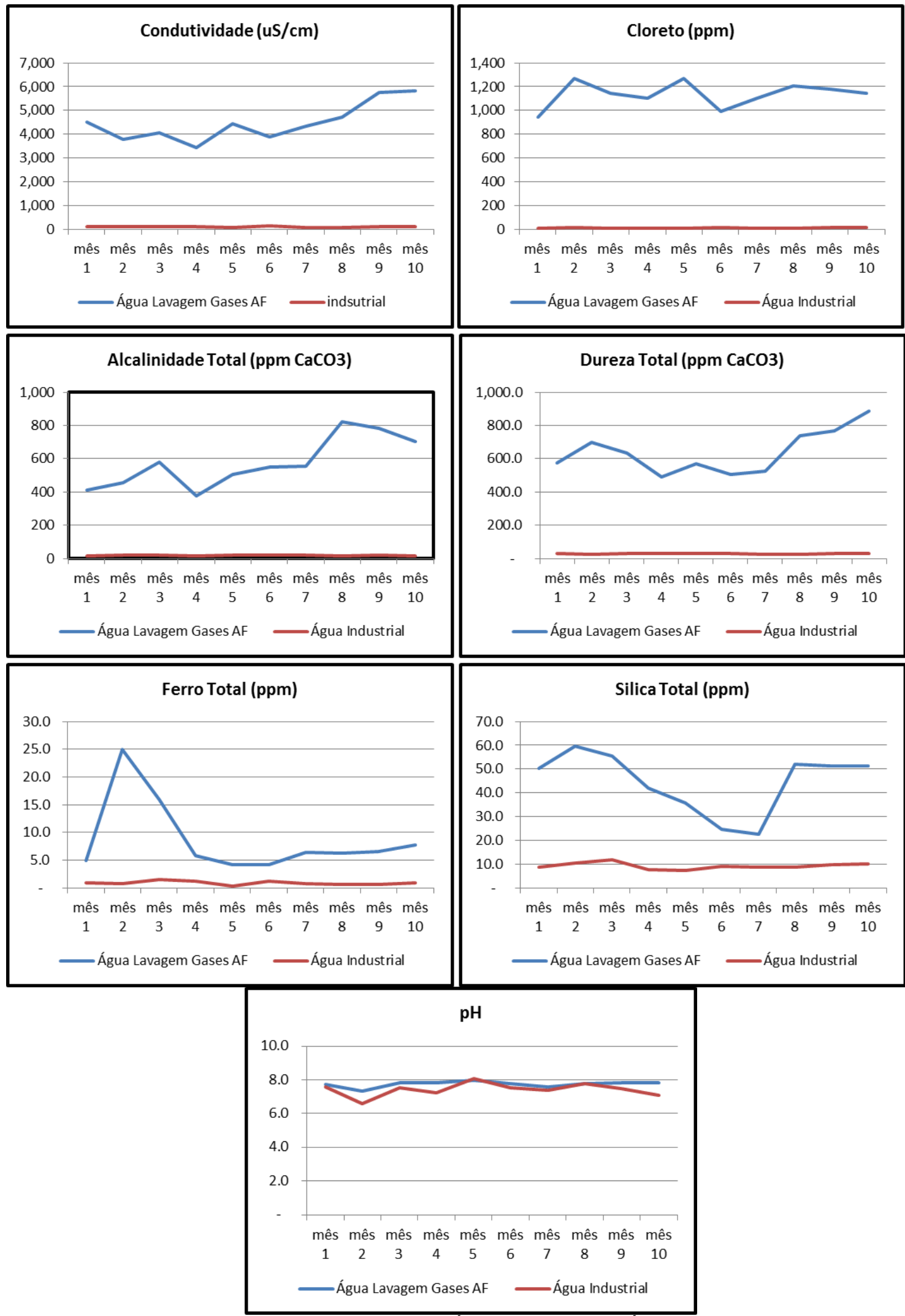

Figura 2. Comparativo de qualidade de água: Água Industrial vs Água Lavagem de Gases AF 
Assim, quando a água de lavagem de gases era utilizada, aumentava o índice de adesão nas paletas do stator blade da turbina devido à maior concentração de elementos depositantes (ferro e cálcio). Passou-se, então, a aplicar o polímero de alto estresse em conjunto com um estabilizante de dureza, cada vez que esta água era utilizada, visando reduzir esta deposição.
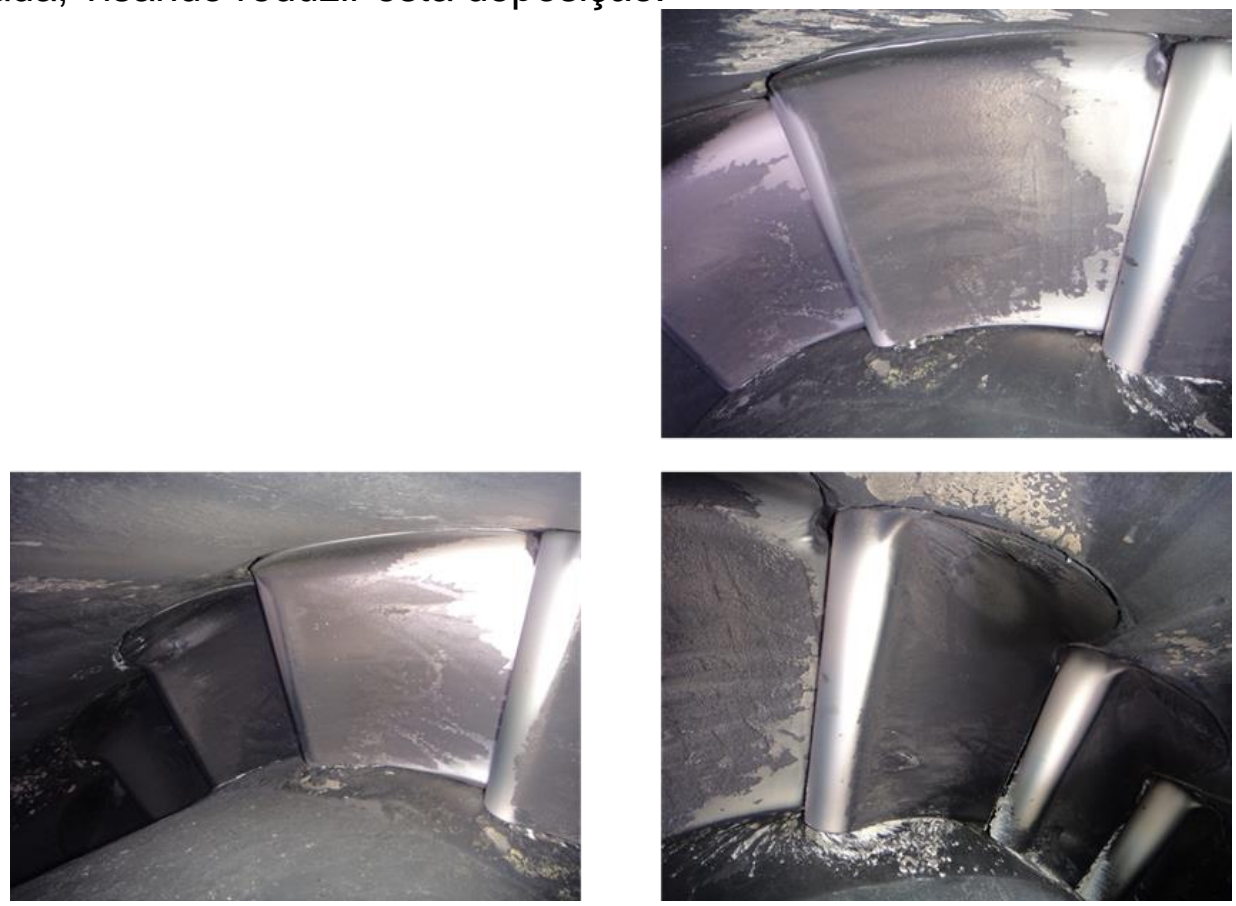

Figura 3. Fotos das palhetas do stator blade

As condições operacionais desta TRT são as seguintes:

Tabela 1. Dados operacionais TRT

\begin{tabular}{|l|l|}
\hline TRT - Design Rated Cap (MW) & 20.20 \\
\hline TRT - Specific PW Generation (KWH/THM) (Design/Actual) & $46.2 / 34.3$ \\
\hline TRT - Current/Actual Generation (MW) & $15(\Delta 14 \sim 17)$ \\
\hline TRT Type -- Axial / Radial turbine? & Axial \\
\hline
\end{tabular}

Para avaliação da performance do programa químico, foram avaliadas as seguintes variáveis do sistema:

- Vazão normalizada de gás do alto forno;

- Output de potência da turbina;

A vazão normalizada de gás de alto forno leva em consideração, além da vazão, a temperatura e pressão, eliminando assim a influência destas duas variáveis de processo na avaliação da aplicação do programa químico.

Com vazão normalizada de gás e potência da turbina, foi possível calcular a geração específica de energia da turbina, com unidade de medida em $\mathrm{KW} / \mathrm{Ndam}^{3}$. Desta forma, a performance da turbina pode ser avaliada de forma direta, onde a queda de geração específica representa uma perda de performance do equipamento. 
Após cada parada da turbina, a curva de geração específica tem um período de estabilização, que para o caso em estudo foram considerados os 20 primeiros dias de campanha pós parada/manutenção, pois após este período inicia-se a tendência a queda do indicador em questão.

Para avaliar o rendimento ao longo da campanha, a média acumulada da geração específica dia a dia foi comparada à geração especifica média dos 20 primeiros dias.

Esta comparação permite avaliar a queda de rendimento da turbina ao longo da campanha e sua tendência.

Foram monitoradas diversas campanhas da turbina, e para efeitos de comparação e avaliação da eficácia do programa químico foram comparadas duas campanhas subsequentes, a primeira sem nenhum tipo de tratamento químico na agua de spray e a segunda com aplicação do programa químico aqui descrito.

\section{RESULTADOS E DISCUSSÃO}

A aplicação do programa químico na agua de spray da turbina resultou em ganho direto de eficiência, redução de custo operacional com aumento da geração de energia e possibilidade de economia de água industrial com o reuso de água de lavagem de gases - de pior qualidade - para o spray.

No item de eficiência e redução de custo operacional, foi observado um aumento direto de $50 \%$ na geração de energia da turbina durante a campanha na qual foi aplicado o programa químico com polímero de resistência a altos padrões de estresse.

É importante ressaltar que com o passar dos meses vem ocorrendo um aumento na taxa de pó no gás do alto forno. Isto se deve a uma série de fatores, como qualidade de matéria-prima dos altos-fornos, taxa de finos no alto-forno, entre outros. $\mathrm{O}$ principal impacto deste aumento da taxa de pó no gás, para a aplicação aqui discutida, é um aumento da adesão de material nas pás do stator blade e da turbina. Mesmo com essas variáveis adversas à eficiência da turbina o programa químico se mostrou bastante eficaz e estável durante sua aplicação. 


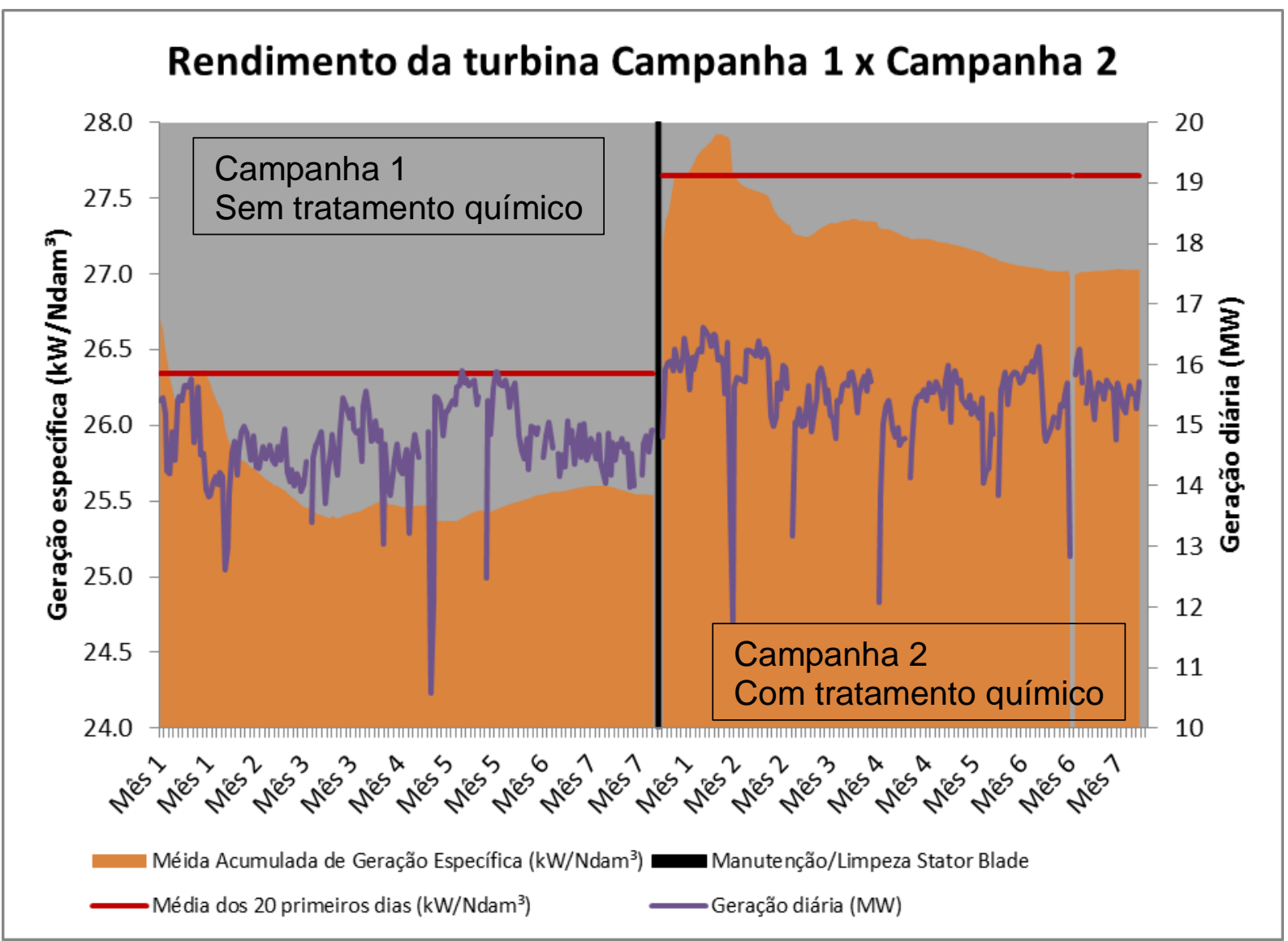

Figura 4. Gráfico comparativo de campanhas com e sem tratamento químico

A figura 4. Apresenta a evolução das variáveis analisadas durante as duas campanhas. Ambas as campanhas tiveram duração aproximada de 7 meses. A área cinza do gráfico entre as curvas vermelha e alaranjada representam a perda de rendimento da turbina ao longo da campanha, visto que a geração específica média de energia tende a cair e se afastar da média dos 20 primeiros dias. 


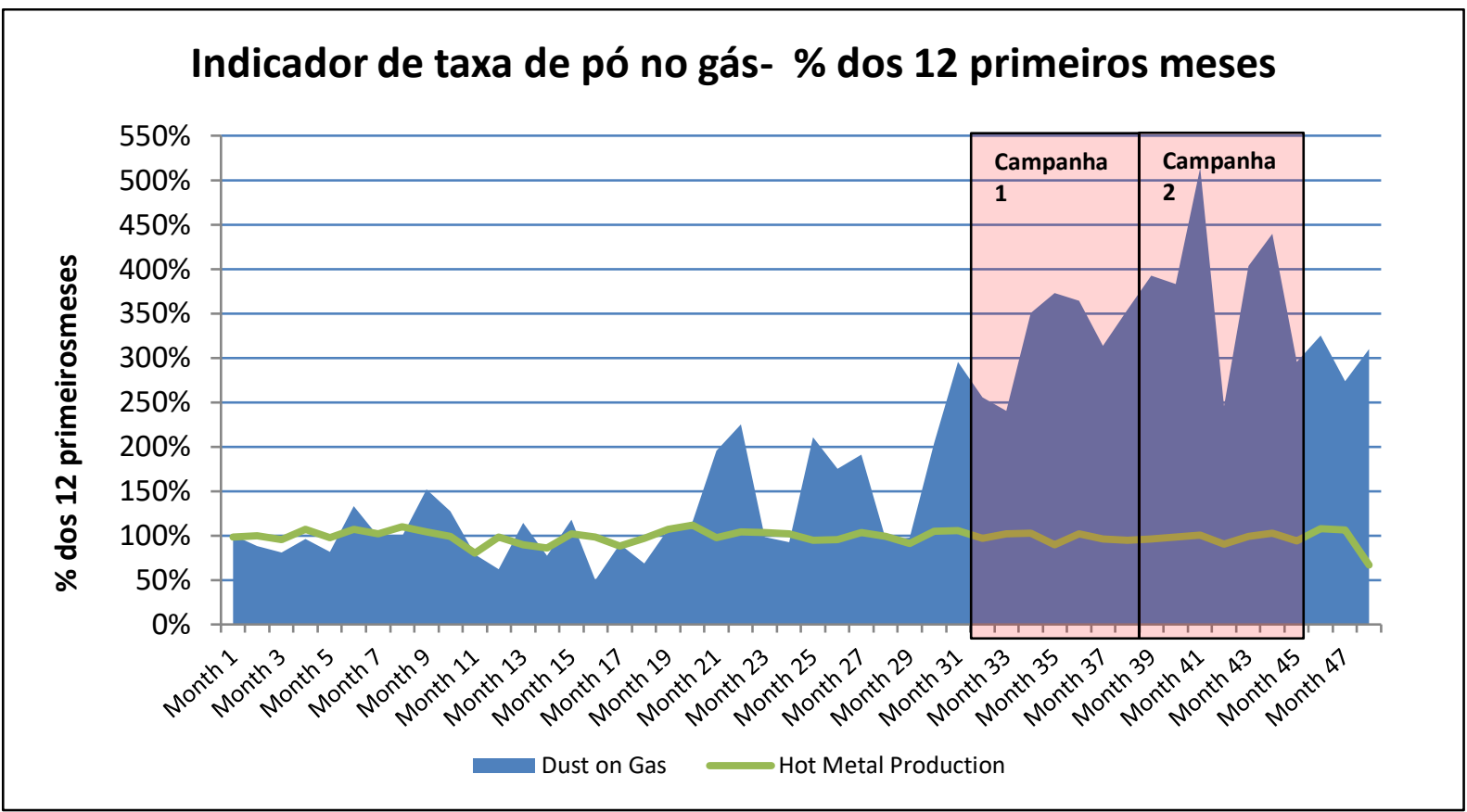

Figura 5. Gráfico do acompanhamento da evolução da taxa de pó no gás de alto-forno

Conforme indicado a taxa de pó acarreta em um maior potencial de deposição de material na turbina, e como podemos observar na figura 5., durante o período de teste do programa químico pode-se observar um aumento médio de $380 \%$ na taxa, com picos acima de $400 \%$.

Mesmo com o uso de água proveniente da lavagem de gás do alto forno e com a taxa de pó do gás com valores bastante altos, houve uma melhora de $52 \%$ no rendimento da turbina.

Tabela 2. Título da tabela sem ponto final

\section{Perda acumulada de geração durante a campanha}

\section{Campanha 1 - sem tratamento químico \\ Campanha 2 - com aplicação de \\ $1943 \mathrm{MW}$ \\ $941 \mathrm{MW}$}

Conforme indicado na tabela 1 , a melhoria de $52 \%$ no rendimento acarretou em um aumento direto de 1002 MW de geração durante a campanha com a aplicação do polímero de alto estresse reduzindo a perda de rendimento da turbina.

\section{CONCLUSÃO}

A implementação do tratamento químico com polímero dispersante com resistência a altos níveis de estresse aumentou o rendimento da turbina de topo do alto forno, sua confiabilidade e provou ser uma aplicação com impacto ambiental importante com a possibilidade de reutilização de água para o spray da turbina. Esta aplicação pode ajudar as siderúrgicas que utilizam a tecnologia de turbina de topo a melhorarem o desempenho da turbina aumento sua geração ou reduzindo sua perda de performance ao longo da campanha e reduzindo o uso de água nobre da usina.

\section{REFERÊNCIAS}

